\title{
Evaluación de las acciones de la educación preventiva contra los efectos nocivos por la exposición prolongada a la radiación solar (uv-b), en las instituciones educativas de la Provincia De Huaraz.
}

Evaluation of the actions of preventive education against the harmful effects for prolonged exposure to solar radiation (uv-b), in the educational institutions in the Province of Huaraz.

${ }^{1}$ Rosa Rodríguez Anaya ${ }^{\mathrm{a}}{ }^{1}$ Yrma Minaya Salinas ${ }^{\mathrm{a}},{ }^{1}$ Liszeth Cerna Ruiz ${ }^{\mathrm{a}},{ }^{1}$ Edward S. Sánchez Rodríguez ${ }^{\mathrm{b}} \mathrm{y}$ ${ }^{1}$ Karen Y. Sallari Gutierrez ${ }^{\mathrm{b}}$

\section{RESUMEN}

La presente investigación tiene como objetivo general evaluar las acciones de la educación preventiva contra los efectos nocivos por la exposición prolongada a la radiación solar (UVB), en las Instituciones Educativas de la Provincia de Huaraz 2015; para lo cual se determinó la Radiación UV-B en la provincia de Huaraz de enero a diciembre del 2015 estableciendo si esta radiación supera los LMP, debiéndose así implementar acciones de Educación Preventiva ante los efectos negativos de radiación UV-B. La metodología utilizada es propia de una investigación aplicada teniendo como muestra los alumnos de las aulas seleccionadas del segundo grado del nivel primario de las Institución Educativa Jorge Basadre Grohmann, La Libertad, Johannes Gutenberg y N 86029 de Huanchac de la provincia de Huaraz; utilizándose encuestas y documentos de las entidades involucradas en el tema, se aplicó una lista de chequeo y encuestas en dos grupos: control y experimental, así como el desarrollo de capacitaciones y uso de materiales para evaluar las variables establecidas.

Los resultados muestran el promedio anual la radiación UV-B en la Provincia de Huaraz en horarios de 9:00 a 16:00 horas, con niveles de riesgo alto, muy alto y extremo, mostrando lo perjudicial para la salud de la población y sobre todo en los niños los efectos nocivos de la radiación solar (UV-B); se demuestra que existe una diferencia significativa en las acciones de la educación preventiva en las Instituciones Educativas por la falta de capacidades, conocimientos, actitudes y valores para desarrollar la Educación Preventiva en los alumnos del segundo grado de las Instituciones Educativas en estudio. Asimismo, las capacitaciones influyen positivamente en los niños generando un cambio de actitud e incrementan sus conocimientos para protegerse ante los efectos negativos de la radiación solar(UV-B); por lo tanto se debe tomar obligatoriamente acciones contra estos efectos especialmente en los alumnos de las Instituciones Educativas por ser el grupo más vulnerable de la población en general.

Palabras clave: educación en la radiación UV-B, prevención ante los efectos nocivos por la radiación $\mathrm{UV}-\mathrm{B}$, acciones preventivas contra la radiación $\mathrm{UV}-\mathrm{B}$ 


\begin{abstract}
The present study general objective is to evaluate the actions of preventive education against the harmful effects for prolonged exposure to solar radiation (UV-B), in the educational institutions in the province of Huaraz 2015; to which determined the UV-B radiation in the province of Huaraz from January to December of 2015 establishing if this radiation exceeds the LMP, and should be so implement actions of prevention education against the negative effects of UV-b radiation The methodology used is typical of an applied research resulting in sample students in classrooms selected from the second grade of the primary level of the educational institution, Jorge Basadre Grohmann, La Libertad, Johannes Gutenberg and $\mathrm{N}^{\circ}$ 86029 of The province of Huaraz Huanchac; using surveys and documents of entities involved in the issue, applied a Checklist and surveys in two groups: control and experimental, as well as skills development and use of materials to assess the variables established.

Results show the average annual UV-B radiation in the province of Huaraz at times from 9:00 to 16:00 hours, with very high and extreme high risk levels, showing the harmful effects of solar radiation (UV-B); s as detrimental to the health of the population and especially children and it shows that there is a significant difference in the actions of preventive education in schools due to the lack of skills, knowledge, attitudes and values to develop preventive education in the students of the second degree of educational institutions in the study. Also the skills positively influencing children creating a change in attitude and increase their knowledge to protect themselves against the negative effects of the solar radiation (UV-B); therefore it should be mandatory actions against these effects particularly in the students of educational institutions for being the most vulnerable group of the population in general.
\end{abstract}

keyword: education in UV-B radiation, prevention before the harmful effects by radiation UV-B, UV-B radiation preventive actions

${ }^{1}$ Universidad Nacional “Santiago Antúnez de Mayolo”. Huaraz, Perú

${ }^{a}$ Lic. en Educación, ${ }^{\mathrm{b}}$ Ing. Ambiental 


\section{INTRODUCCIÓN}

La acción de la radiación solar en el ser humano posee unos efectos que condicionan su salud. La información científica demuestra que la exposición excesiva y/o acumulada de radiación ultravioleta de fuentes naturales o artificiales produce efectos dañinos a corto y largo plazo. Cuando nos exponemos al sol Gutiérrez (2009) indica que además de tener en cuenta la intensidad y la frecuencia de la radiación, un factor determinante es la susceptibilidad y el grado de tolerancia propios de cada persona que se expone a la radiación solar; por lo tanto el sol, tomado con moderación, ejerce efectos beneficiosos para el organismo, pero una exposición incontrolada, sin protección y demasiado prolongada, puede ocasionar graves consecuencias para la salud. Sabemos que la radiación UVA, pese a no producir quemaduras solares, a largo plazo es responsable del envejecimiento cutáneo prematuro de la piel conocido como foto envejecimiento (BIOSALUD, 2009).

Reconociendo estos datos nos permite plantear una investigación que tiene como objetivo general evaluar las acciones de la educación preventiva contra los efectos nocivos por la exposición prolongada a la radiación solar (UV-B), en las Instituciones Educativas de la Provincia de Huaraz 2015, generando acciones contra estos efectos especialmente en los alumnos de las Instituciones Educativas por ser el grupo más vulnerable de la población en general; en tal sentido Ríos (2008) menciona que la radiación que pasa a través de la capa de ozono puede causar los siguientes problemas, especialmente en las personas que están mucho tiempo al sol:

- Cáncer de piel y otros trastornos cutáneos.

- Cataratas y otros trastornos de la vista.

- Inhibición del sistema inmunitario.

Debido a estos efectos adversos, es importante limitar la exposición a la radiación ultravioleta y protegerse al estar trabajando, jugando o haciendo ejercicios al aire libre.

Esta investigación plantea a su vez objetivos específicos enfocados a recopilar y determinar los niveles de radiación solar (UV-B) que ha recibido la provincia de Huaraz de Enero a Diciembre del 2015; las acciones de la educación preventiva contra los efectos nocivos por la exposición prolongada a la radiación solar (UV-B) en los alumnos del segundo grado de las Instituciones Educativas Jorge Basadre Grohmann, La Libertad, Johannes Gutenberg y Nº 86029 de Huanchac; asimismo fortalecer capacidades, conocimientos, actitudes y valores para desarrollar la Educación Preventiva contra los efectos nocivos por la exposición prolongada a la radiación solar (UV-B) en los alumnos y determinar las acciones necesarias para la protección contra los efectos nocivos por la exposición prolongada a la radiación 
solar (UV-B); considerando que el Instituto Nacional del Cáncer (2014) informa que muchos de los efectos de la exposición al sol no se manifiestan hasta la edad adulta, los estudios médicos recientes muestran que es muy importante proteger a los niños y adolescentes de la sobreexposición a la radiación ultravioleta. La mayor parte de la exposición al sol de una persona tiene lugar antes de los 18 años de edad, y los estudios realizados indican claramente que hay una relación entre la exposición al sol a una edad temprana y el cáncer de piel en la edad adulta.

Es de suma importancia reconocer que los resultados de esta investigación promueven la Educación Preventiva contra los efectos nocivos por la exposición prolongada a la radiación solar en los alumnos del segundo grado de las Instituciones Educativas en estudio, y se generan por la percepción y la falta de información de los actores involucrados.

\section{MATERIALES Y MÉTODOS}

El tipo de estudio corresponde a una investigación causal explicativa. El diseño de investigación aplicado es cuasi experimental, de dos grupos no equivalentes conocido como preprueba posprueba y grupo control; la población son los alumnos del segundo grado del nivel primario de las Instituciones Educativas Jorge Basadre Grohmann, La Libertad, Johannes Gutenberg y Nº 86029 de Huanchac, y la muestra conformada por los alumnos de las aulas seleccionadas del segundo grado del nivel primario de las mencionadas Instituciones Educativas.

Respecto a los instrumentos de recolección de la información se aplicó una encuesta con el propósito de evaluar la importancia de los indicadores de las variables de investigación, asimismo se utilizó un instrumento adicional que fue la entrevista, para conocer la opinión de los alumnos y personal docente de las instituciones educativas en estudio acerca de la educación preventiva y su adecuada aplicación; luego se utilizó la observación como un registro visual de lo que ocurre en una situación real, se recogen los datos mediante una lista de chequeo, que son parte de una metodología empleada para determinar acciones de la educación preventiva contra los efectos nocivos por la exposición prolongada a la radiación solar (UV-B) que incluye una programación de actividades de intervención en el grupo control y experimental.

La información obtenida de la encuesta realizada, sirvió para diseñar la estructura estadística del proyecto de investigación, pero con los datos recolectados, se confirmó los problemas, la validez de la hipótesis y el cumplimiento de los objetivos a través de los cuadros estadísticos para analizarlos e interpretarlos desde el punto de vista de la educación preventiva. Para la comprobación de la hipótesis se utilizó la $\mathrm{T}$ de Student para muestras relacionadas. 


\section{RESULTADOS}

A. Determinación los niveles de radiación solar (UV-B) que ha recibido la provincia de Huaraz de enero a diciembre del 2015

Se recabaron dos tipos de información para determinar los niveles de Radiación
Ultra Violeta - B como el monitoreo de radiación ultavioleta y la radiación solar global para determinar Correlación entre la Información de Radiación Solar Global y Radiación Ultra Violeta - B del 29-012015 al 03-02-2015 en la Estación Meteorológica de Shancayan, confluyendo en el siguiente resultado:

Tabla $\mathbf{N}^{0}$ 01. Promedio Anual aplicando la Correlación entre la Información de Radiación Solar Global $\left(\mathrm{W} / \mathrm{m}^{2}\right)$ y Radiación Ultra Violeta $-\mathrm{B}\left(\mathrm{W} / \mathrm{m}^{2}\right)$ de Enero a Diciembre del 2015.

\begin{tabular}{cccccc}
\hline $\begin{array}{l}\text { Indice } \\
\text { UV-B }\end{array}$ & $\begin{array}{c}\text { Nivel de Riesgo } \\
\text { Radiación UV-B }\end{array}$ & $\begin{array}{c}\text { Rangos (W/m2) } \\
\text { Radiación UV-B }\end{array}$ & $\begin{array}{c}\text { Rangos (W/m2) } \\
\text { Radiación UV-B }\end{array}$ & $\begin{array}{c}\text { Porcentaje } \\
\text { Promedio de } \\
\text { Indice UV-B (\%) }\end{array}$ & Hora \\
\hline $1 .-2$ & Minimo & 60.00 & 0.676267 & $5.49 \%$ & $07-08$ y $16.00-19.00$ \\
$3 .-5$ & Bajo & 150.00 & 0.471018 & $6.33 \%$ & $08.00-09.00$ \\
$6 .-8$ & Moderado & 400.00 & 0.251312 & $16.20 \%$ & $09.00-10.00 \mathrm{y}$ \\
$9 .-11$ & Allto & 900.00 & 0.069664 & $32.74 \%$ & $14.00-16.00$ \\
$12 .-14$ & Muy Alto & 1300.00 & 0.012707 & $29.64 \%$ & $10.00-11.00$ \\
$>14$ & Extremo & 1300.00 & 0.059218 & $9.60 \%$ & $1200-12.00$ \\
\hline
\end{tabular}

Fuente: Elaborado por el Ing. Rafael Figueroa Tauquino

De la Tabla $\mathrm{N}^{\circ} 1$ se observa que:

Después de determinando el nivel de riesgo de la radiación UV-B y los horarios de mayor incidencia por meses de Enero a Diciembre del 2015, se determinó el promedio anual aplicando la Correlación entre la Información de Radiación Solar Global $\left(\mathrm{W} / \mathrm{m}^{2}\right)$ y Radiación Ultra Violeta - B $\left(\mathrm{W} / \mathrm{m}^{2}\right)$ de Enero a Diciembre del 2015.

El promedio anual de la radiación UV-B con un índice de 9-11 Nivel Riesgo Alto en los intervalos de 10:00 a 11:00 horas, con un índice de 12-14 Nivel Riesgo Muy Alto de 11:00 a 12:00 horas, con un índice 6-8 Nivel Riesgo Moderado de 9:00 a 10:00 horas y 14:00 a 16:00 horas; y con un índice $>14$ Nivel Riesgo Extremo de 12:00 a 14:00 horas.

Tabla $N^{\circ}$ 02. Determinación del Nivel de Riesgo de la Radiación UV-B de Enero a Diciembre del 2015 en la Provincia de Huaraz.

\begin{tabular}{cccc}
\hline \multicolumn{4}{c}{ Nivel de Riesgo de la Radiación UV-B } \\
\hline Mes & Alto (\%) & Muy Alto (\%) & Extremo (\%) \\
ENE & 21.58 & 29.47 & 19.47 \\
FEB & 23.55 & 30.52 & 11.63 \\
MAR & 21.57 & 29.41 & 15.69 \\
ABR & 27.46 & 31.5 & 10.69 \\
MAY & 34.29 & 32.86 & 1.14 \\
JUN & 66.07 & 9.61 & 0.60 \\
JUL & 55.75 & 22.71 & 0.59 \\
AGO & 42.12 & 33.52 & 2.58 \\
SEP & 27.20 & 35.69 & 7.93 \\
OCT & 23.97 & 37.19 & 12.12 \\
NOV & 25.00 & 32.67 & 15.34 \\
DIC & 24.38 & 30.47 & 17.45 \\
\hline
\end{tabular}

Fuente: Elaborado por el Ing. Rafael Figueroa Tauquino 


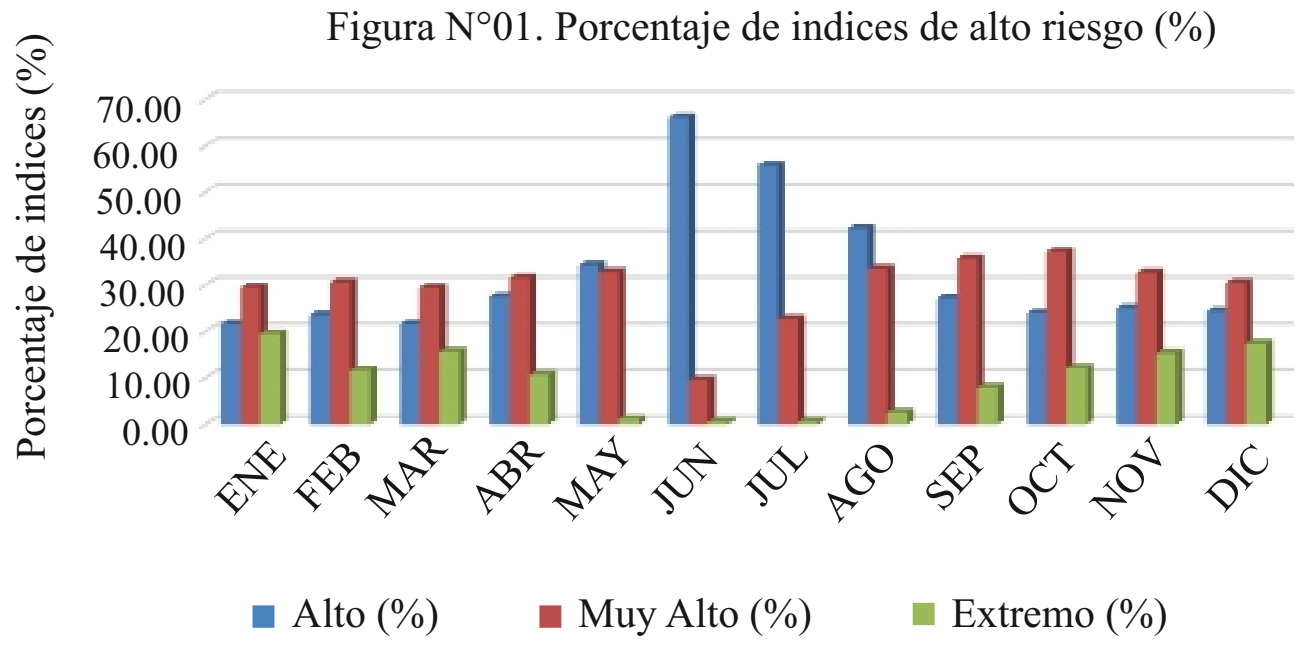

Fuente: Elaborado por el Ing. Rafael Figueroa Tauquino

De la Tabla $\mathrm{N}^{\circ} 1$ y Figura $\mathrm{N}^{\circ} 01$ se observa que:

En los meses de Mayo, Junio, Julio y Agosto predomina una Nivel de Riesgo de radiación UV-B Alto a nivel de la Provincia de Huaraz, entre los meses de Setiembre a Diciembre y Enero a Abril predomina el Nivel de Riesgo Muy Alto intensificándose en los meses de Setiembre, Octubre y Noviembre.

A. Acciones de la Educación Preventiva contra los efectos nocivos por la exposición prolongada a la radiación solar (UV-B), en los alumnos del segundo grado de las Instituciones Educativas Jorge Basadre Grohmann, La Libertad, Johannes Gutenberg y $N^{\circ}$ 86029 de Huanchac.
En el presente ítem se muestran los resultados obtenidos de la comparación de los puntajes sobre Acciones de la Educación Preventiva Contra los Efectos Nocivos por la Exposición Prolongada a la Radiación Solar (UV-B). Se recopilaron los datos en las cuatro Instituciones Educativas del 07 al 11 de Setiembre del 2015 mediante observación y una lista de chequeo que da una puntuación y con el permiso de las autoridades de las instituciones educativas. La finalidad de esta toma de datos fue conocer las realidades de las instituciones educativas de forma independiente y comprarlas entre sí. 


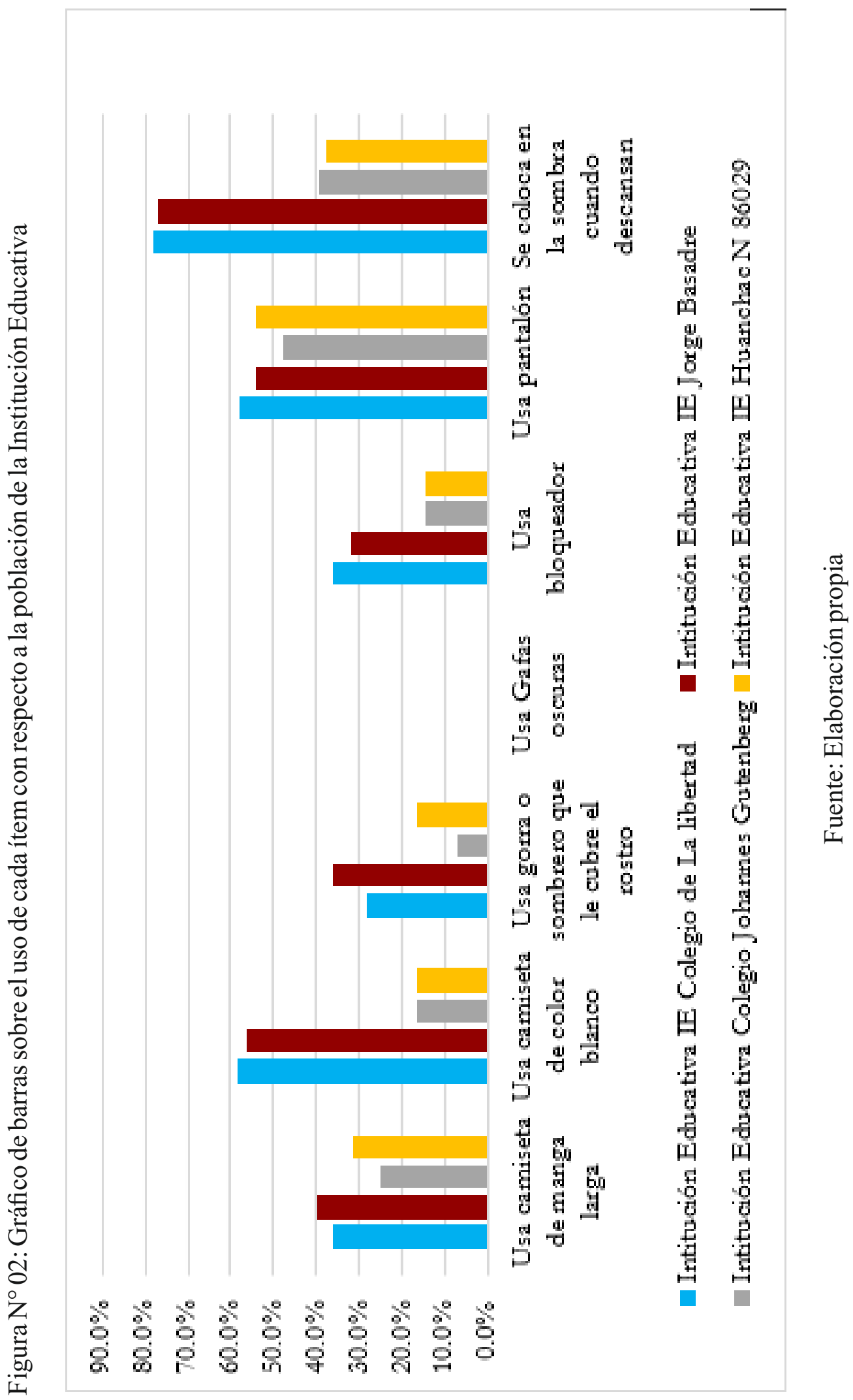


De la figura $\mathrm{N}^{\circ} 02$ se observa que:

El ítem "Se coloca en la sombra cuando descansan" es el que mejor porcentaje de las cuatro instituciones superando el $70 \%$ en el caso de la IE Colegio la Libertad y la IE Jorge Basadre, mientras el ítem "usa gafas oscuras" tiene un porcentaje de cero en todas las instituciones educativas.

La mayoría de los ítems no supera el 40\% mientras que usa pantalón es el más homogéneo.

\section{DISCUSIÓN}

Con la finalidad de comprobar la Hipótesis General se determinó los Niveles de Radiación e Índice UV-B para la Provincia de Huaraz empleando los datos de monitoreo de 6 días, determinando una ecuación de correlación entre la Información de Radiación Solar Global (W/m2) y Radiación Ultra Violeta - B (W/m2) del 29-01-2015 al 03-02-2015 en la Estación Meteorológica de Shancayan. La Ecuación de correlación logarítmica nos permitió proyectar de Enero a Diciembre la Radiación UV B y determinar los promedio mensual y anual del 2015 así como los horarios de mayor incidencia y riesgo en función a la tabla establecida por la OMS de Índice y Riesgo UV solar mundial, con el propósito de evaluar las acciones de educación preventiva contra los efectos nocivos por la exposición prolongada de radiación UV-B en las Instituciones Educativas de la Provincia de Huaraz.
En la provincia de Huaraz se determinó que en promedio anual la radiación UV-B tiene un índice de 9-11 en los intervalos de 10:00 a 11:00 horas, con un índice de 1214 en los intervalos de 11:00 a 12:00 horas, con un índice 6-8 en los intervalos de 9:00 a 10:00 horas y 14:00 a 16:00 horas; y con un índice $>14$ en el intervalo de 12:00 a 14:00 horas.

En tal sentido se tiene que implementar acciones de Educación Preventiva ante los efectos negativos de radiación UB-V. (Ver Tabla $\left.\mathrm{N}^{\circ} 01\right)$.

Así mismo se determinó para la provincia de Huaraz el promedio anual de la radiación UV-B con Nivel Riesgo Alto en los intervalos de 10:00 a 11:00 horas, con Nivel Riesgo Muy Alto de 11:00 a 12:00 horas, Nivel Riesgo Moderado de 9:00 a 10:00 horas y 14:00 a 16:00 horas y con Nivel Riesgo Extremo de 12:00 a 14:00 horas. Debiendo implementar acciones de Educación Preventiva ante los efectos negativos de radiación UV-B. (Ver Tabla $\mathrm{N}^{\circ} 01$ ).

En los meses de Mayo, Junio, Julio y Agosto predomina una Nivel de Riesgo de radiación UV-B Alto a nivel de la Provincia de Huaraz que amerita la implementación más intensiva de acciones preventivas contra los efectos nocivos de la radiación solar (UV-B). Este efecto es debido a la cercanía del sol a la tierra Afelio entre los meses de Mayo a Agosto y Perihelio entre los meses de Setiembre a Diciembre y Enero a Abril donde predomina el Nivel de Riesgo Muy Alto especialmente los meses de 
Setiembre, Octubre y Noviembre donde las acciones preventivas contra los efectos de la radiación solar (UV-B) deberán ser todavía más intensivos y eficientes.

El análisis descriptivo de los datos obtenidos tanto con la lista de chequeo muestra cómo están distribuidos los datos, es decir el mínimo, máximo, promedio, desviación típica (que nos indica la variabilidad de los datos de cada grupo y que resultaron ser homogéneos con ligeras variaciones) tanto Antes $y$ Después en ambos grupos, control y experimental. Aquí se nos muestra los promedios que luego serán contrastados en las pruebas de hipótesis estadísticas de T-Student.

La prueba de hipótesis de T-Student nos demuestra que hay diferencias entre las Instituciones Educativas de la provincia de Huaraz siendo la I.E. Johannes Gutemberg quien tiene un menor promedio de puntuación con respecto a los otros tres colegios, además se observó que la I.E. La Libertad y la I.E. Jorge Basadre son más homogéneos en sus promedios por encima de la I.E. Huanchac $\mathrm{N}^{\circ}$ 86029. Por lo tanto, se confirma que para la hipótesis específica 1 que Existe una diferencia significativa en las acciones de la educación preventiva en las Instituciones Educativas de la Provincia de Huaraz.

La prueba de hipótesis de T-Student de Antes y Después, nos demuestra que estas variables han influido en el resultado final de haciendo que haya una mejora estadísticamente significativa en el grupo experimental después de aplicada la metodología de las ACCIONES DE LA EDUCACIÓN PREVENTIVA CONTRA LOS EFECTOS NOCIVOS POR LA EXPOSICIÓN PROLONGADA A LA RADIACIÓN SOLAR (UV-B), es decir que el grupo control se mantuvo antes y después ya que al aplicar las pruebas no se encontró diferencia significativa entre los promedios de los puntajes.

Por otro lado en el grupo experimental la situación fue diferente ya que este grupo si aumento su puntuación en promedio, $\mathrm{y}$ fue demostrado que si hay una diferencia significativa entre la medición antes de la aplicación de las ACCIONES DE LA EDUCACIÓN PREVENTIVA CONTRA LOS EFECTOS NOCIVOS POR LA EXPOSICIÓN PROLONGADA A LA RADIACIÓN SOLAR (UV-B), y la medición después de estas, demostrando así que esta metodología influye positivamente en el puntaje los niños confirmando la hipótesis especifica 2 planteada en la investigación.

\section{CONCLUSIONES}

En la provincia de Huaraz tenemos en promedio anual la radiación UV-B con un índice de 9-11 Nivel Riesgo Alto en los intervalos de 10:00 a 11:00 horas, con un índice de 12-14 Nivel Riesgo Muy Alto de 11:00 a 12:00 horas, con un índice 6-8 Nivel Riesgo Moderado de 9:00 a 10:00 horas y 14:00 a 16:00; y con un índice >14 
Nivel Riesgo Extremo de 12:00 a 10:00 horas. En tal sentido se tiene que implementar acciones de Educación Preventiva ante los efectos negativos de radiación UV-B.

En los meses de Mayo, Junio, Julio y Agosto predomina una Nivel de Riesgo de radiación UV-B Alto a nivel de la Provincia de Huaraz, entre los meses de Setiembre a Diciembre y Enero a Abril predomina el Nivel de Riesgo Muy Alto intensificándose en los meses de Setiembre, Octubre y Noviembre. (Ver Figura $\mathrm{N}^{\circ} 02$ ).

En la provincia de Huaraz el Nivel de Riesgo de la Radiación Solar (UV-B) durante todo el año es Alto, Muy Alto y Extremo en el intervalo de 9:00 a 16:00 horas por lo cual se debe tomar obligatoriamente acciones contra los efectos de la radiación solar (UV-B) especialmente en los alumnos de las Instituciones Educativas por ser el grupo más vulnerable de la población en general.

Se demuestra que existe una diferencia significativa en las acciones de la educación preventiva en las Instituciones Educativas de la Provincia de Huaraz, afirmando así la Hipótesis Específica 1 de la presente tesis. Esta diferencia se evidencia de forma más marcada en las Instituciones Educativas de Jorge Basadre y La Libertad con el Johannes Gutemberg y Huanchac $N^{\circ} 86029$. Es decir las dos primeras (Jorge Basadre y La Libertad) tienen características similares debido a ser Instituciones que alberga numerosa cantidad de alumnos, con Infraestructura adecuada y más estricto de las directiva y normativa emitida por el Ministerio de Educación y las dos últimas Johannes Gutemberg y Huanchac No 86029 una por ser una Institución privada y la otra de zona rural sus condiciones son diferentes, su infraestructura es mínima y no existe mucha rigurosidad en el cumplimiento de las directivas y normativa emitida por el Ministerio de Educación.

Así mismo se afirma la Hipótesis Específica 2 y se demuestra que existen falta de capacidades, conocimientos, actitudes y valores para desarrollar la Educación Preventiva contra los efectos nocivos por la exposición prolongada a la radiación solar en los alumnos del segundo grado de las Instituciones Educativas en estudio, y se generan por la percepción y la falta de información de los actores involucrados. Después de la prueba de hipótesis realizada podemos concluir que después de aplicar la metodología de las ACCIONES DE LA EDUCACIÓN PREVENTIVA CONTRA LOS EFECTOS NOCIVOS POR LA EXPOSICIÓN PROLONGADA A LA RADIACIÓN SOLAR (UV-B) mediante capacitaciones, charlas, videos, gigantografías, etc. Influye positivamente en los niños y empieza a cambiar de actitud e incrementar sus conocimientos en su cuidado ante los efectos negativos de la radiación solar (UV-B). 
Habiendo determinado que en la provincia de Huaraz los Niveles de Riesgo de la Radiación Solar (UV-B) predominantemente son Altos, Muy Altos y Extremos en el intervalo de 9:00 a 16:00 horas se debe inculcar de manera más intensiva actividades dirigidas a prevenir los efectos nocivos radiación solar, promocionar estilos de vida saludable, limitar la exposición a la radiación solar y la exposición usando medidas de protección (mantenerse bajo la sombra, usar bloqueador solar, ropa adecuada que proteja la piel lentes oscuros con protección UV, pantalón y camisa de manga larga, sombrilla).

\section{REFERENCIAS BIBLIOGRÁFICAS}

(11 de Febrero de 2014). Obtenido de Instituto Nacional del Cáncer: http://www.cancer.gov/espanol/pdq/ tratamiento/piel/Patient/page1\#Key point2.

BIOSALUD. (14 de enero de 2009). Obtenido de BIOSALUD: http://www.biosalud.org/inde2.php? $\mathrm{sec}=2 \& \mathrm{id}=140$

GUTIERREZ, C. (2009). Campaña de educación, prevención y detección temprana del cáncer de piel y melanoma. Informe Final "El Día del Lunar", 7-9.

GUTIERREZ, C. (2011). Campaña de educación, prevención y detección temprana del cáncer de piel y melanoma. Informe Final "El Día del Lunar", 9-11.
RAMOS, W. C., \& VENEGAS, D. R. (2013). Análisis de la Situación del Cáncer en el Perú. Lima: EdukPerú.

RIOS, D. (2008). Conocimiento sobre efectos nocivos de la radiación solar en Arequipa. Ica: Marruecos.

\section{CORRESPONDENCIA}

Rosa D. Rodríguez Anaya rosanaya2012@gmail.com 